International Journal of Applied Mathematics

Volume 32 No. $3 \quad 2019,433-441$

ISSN: 1311-1728 (printed version); ISSN: 1314-8060 (on-line version)

doi: http://dx.doi.org/10.12732/ijam.v32i3.5

\title{
OSCILLATION CRITERIA OF A CLASS OF FRACTIONAL ORDER DAMPED DIFFERENCE EQUATIONS
}

\author{
A. George Maria Selvam ${ }^{1}$, R. Janagaraj ${ }^{1}$ \\ ${ }^{1} \mathrm{PG} \&$ Research Department of Mathematics \\ Sacred Heart College (Autonomous) \\ Tirupattur-635601, Vellore Dt., Tamil Nadu, S. INDIA
}

\begin{abstract}
Herein, we examine the oscillatory behavior of all solutions of a fractional order difference equations with damping term of the form

$$
\Delta^{1+\alpha} u(t)+p(t) \Delta^{\alpha} u(t)+q(t) F[G(t)]=0, \quad t \geq t_{0}>0,
$$

where $G(t)=\sum_{s=t_{0}}^{t-1+\alpha}(t-s-1)^{-\alpha} u(s)$ and $\Delta^{\alpha}$ denotes the Riemann-Liouville difference operator of order $0<\alpha \leq 1$. We arrive at some new sufficient conditions for the oscillation of solutions of fractional order damped difference equations using generalized riccati type transformation technique under suitable conditions.
\end{abstract}

AMS Subject Classification: 39A21, 34A08, 39A10

Key Words: oscillation, fractional order difference equations, Riccati technique, damping term

\section{Introduction}

Riccati type transformations are useful in the investigation of oscillation of solutions of differential/difference equations. Recently, paper [5] dealt with oscillation criteria of fractional differential equations. Motivated basically by this paper, and the cited papers in the references, we aim at obtaining some new oscillation theorems for a class of damped fractional order difference equations

Received: January 14, 2019

(c) 2019 Academic Publications

${ }^{\S}$ Correspondence author 
of the form

$$
\Delta^{1+\alpha} u(t)+p(t) \Delta^{\alpha} u(t)+q(t) F[G(t)]=0, \quad t \geq t_{0}>0,
$$

where $G(t)=\sum_{s=t_{0}}^{t-1+\alpha}(t-s-1)^{-\alpha} u(s)$ and $\Delta^{\alpha}$ is the Riemann-Liouville difference operator of order $0<\alpha \leq 1$. Following assumptions are considered in the discussion of the this paper:

$\left(C_{1}\right) p:\left[t_{0}, \infty\right) \rightarrow R$ is a continuous function with $p(t)<0$.

$\left(C_{2}\right) q:\left[t_{0}, \infty\right) \rightarrow R$ is a continuous function with $q(t) \geq 0$.

$\left(C_{3}\right) f: R \rightarrow R$ is a continuous function with $u f(u)>0$ and there exists a constant $m>0$ such that $\frac{f(u)}{u} \geq m$ for all $u \neq 0$.

The problem of determining the oscillation of solutions of various equations like ordinary differential equations, difference equations, dynamic equations on timescales and functional differential equations has been a very active area of research in the last few decades (see [6], [7]). Recent years have witnessed the study of qualitative properties, especially oscillation of solutions of fractional difference equations (see [1], [2], [4], [9]) and the references therein.

By using Riccati type transformations, we establish some new sufficient conditions for the oscillation of solutions of equation (1). Therefore it is hoped that this paper will contribute to the study of oscillation for fractional order difference equations with damping term.

\section{Preliminaries and Basic Results}

This section introduces basic definitions and some preliminary results of discrete fractional calculus, which will be used throughout this paper.

Definition 1. (see [8]) A solution $u(t)$ of (1) is said to be oscillatory if it is neither eventually positive nor eventually negative; otherwise, it is nonoscillatory. Equation (1) is said to be oscillatory if all its solutions are oscillatory.

Definition 2. (see [1], [3], [7]) Let $\nu>0$. The $\nu$-th fractional sum $f$ is defined by

$$
\Delta^{-\nu} f(t)=\frac{1}{\Gamma(\nu)} \sum_{s=0}^{t-\nu}(t-s-1)^{\nu-1} f(s),
$$


where $f$ is defined for $s \equiv a \bmod (1)$ and $\Delta^{-\nu} f$ is defined for $t \equiv(a+\nu) \bmod$ (1) and $t^{(\nu)}=\frac{\Gamma(t+1)}{\Gamma(t-\nu+1)}$ and $\Delta^{-\nu} f: N_{a} \rightarrow N_{a+\nu}$.

Definition 3. (see [2], [3], [7]) Let $\mu>0$ and $m-1<\mu<m$ where $m$ denotes a positive integer $m=\lceil\mu\rceil$. Set $\nu=m-\mu$. The $\mu$-th fractional difference is defined as

$$
\Delta^{\mu} f(t)=\Delta^{m-\nu} f(t)=\Delta^{m} \Delta^{-\nu} f(t) .
$$

Theorem 4. (see [1], [2]) Let $u(t)$ be a solution of (1) and $G(t)=$ $\sum_{s=t_{0}}^{t-1+\alpha}(t-s-1)^{-\alpha} u(s)$, then $\Delta G(t)=\Gamma(1-\alpha) \Delta^{\alpha} u(t)$.

Theorem 5. (see [5]) Let $\alpha \in(0,1)$, and $t>0$. If $u$ is a solution of (1), then $\left(\Delta^{1+\alpha} u\right)(t)=\Delta\left(\Delta^{\alpha} u\right)(t)$.

Proof. From Definition (3), by considering $\mu=1+\alpha, m=\lceil 1+\alpha\rceil=$ $1+\lceil\alpha\rceil=1$ and $\nu=1-\mu=1-(1+\alpha)=-\alpha$, we have

$$
\left(\Delta^{1+\alpha} u\right)(t)=\Delta \Delta^{-(-\alpha)} u(t)=\Delta\left[\Delta^{\alpha} u(t)\right] .
$$

\section{Main Results}

This section establishes some sufficient conditions of oscillation criteria results and inequalities.

Theorem 6. Suppose that $\left(C_{1}\right)-\left(C_{3}\right)$ hold. If

$$
\lim _{t \rightarrow \infty} \sum_{s=t_{0}}^{t-1}\left[m q(s)-\frac{p^{2}(s)}{4 \Gamma(1-\alpha)}\right]=\infty,
$$

then every solution of equation (1) is oscillatory.

Proof. Suppose that $u(t)$ is a non oscillatory solution of (1). Without loss of generality, we may take $u(t)$ is an eventually positive solution of (1). Then 
$u(t)>0$ and $G(t)>0$ for $t \geq t_{1}>t_{0}$. Let us define the sequence $\omega(t)$ by Riccati transformation as follows:

$$
\omega(t)=-\frac{\Delta^{\alpha} u(t)}{G(t)} \text { for } t \geq t_{1} .
$$

Here, $\omega(t)$ is well defined and satisfies the inequality for $t \geq t_{1}$. It follows that

$$
\Delta \omega(t)=-\Delta\left[\frac{\Delta^{\alpha} u(t)}{G(t)}\right]=\frac{\left[\Delta^{\alpha} u(t)\right] \Delta G(t)}{G(t) G(t+1)}-\frac{\Delta\left[\Delta^{\alpha} u(t)\right]}{G(t+1)} .
$$

Applying Theorem (4) and Theorem (5) to (1), we get

$$
\Delta \omega(t)=\frac{\Gamma(1-\alpha)\left(\Delta^{\alpha} u(t)\right)^{2}}{G(t) G(t+1)}+p(t) \frac{\Delta^{\alpha} u(t)}{G(t+1)}+q(t) \frac{F[G(t)]}{G(t+1)} .
$$

Since $\Delta G(t)$ is a non increasing function, we have

$$
\Delta \omega(t)>\frac{\Gamma(1-\alpha)\left(\Delta^{\alpha} u(t)\right)^{2}}{(G(t))^{2}}+p(t) \frac{\Delta^{\alpha} u(t)}{G(t)}+q(t) \frac{F[G(t)]}{G(t)} .
$$

From $(3)$ and $\left(C_{3}\right)$, we get

$$
\Delta \omega(t)>\Gamma(1-\alpha) \omega^{2}(t)-p(t) \omega(t)+m q(t) .
$$

Let us sum the above expression (4) from $t_{1}$ to $t-1$ on both sides, we obtain

$$
\begin{aligned}
& \sum_{s=t_{1}}^{t-1} \Delta \omega(s)>\sum_{s=t_{1}}^{t-1}\left[\Gamma(1-\alpha) \omega^{2}(s)-p(s) \omega(s)+m q(s)\right] \\
& =\Gamma(1-\alpha) \sum_{s=t_{1}}^{t-1}\left(\left[\omega(s)-\frac{p(s)}{2 \Gamma(1-\alpha)}\right]^{2}+\left[m q(s)-\frac{p^{2}(s)}{4 \Gamma(1-\alpha)}\right]\right) .
\end{aligned}
$$

In view of (2), there exists $t_{2} \geq t_{1}$ such that

$$
\omega(t)>\Gamma(1-\alpha) \sum_{s=t_{1}}^{t-1}\left[\omega(s)-\frac{p(s)}{2 \Gamma(1-\alpha)}\right]^{2} \quad \text { for } \quad t>t_{2} .
$$

Now we take $H_{1}(t)=\Gamma(1-\alpha) \sum_{s=t_{1}}^{t-1}\left[\omega(s)-\frac{p(s)}{2 \Gamma(1-\alpha)}\right]^{2}$, then

$\omega(t)>H_{1}(t)$ for $t \geq t_{1}$. From $H_{1}(t)$ applying the fact that $p(t)<0$, we can 
easily see that,

$$
\begin{aligned}
\Delta H_{1}(t) & =\Gamma(1-\alpha) \Delta\left[\sum_{s=t_{1}}^{t-1}\left[\omega(s)-\frac{p(s)}{2 \Gamma(1-\alpha)}\right]^{2}\right] \\
& >\Gamma(1-\alpha) H_{1}^{2}(t), \quad \text { for } t>t_{1} \\
\Gamma(1-\alpha) & <\frac{\Delta H_{1}(t)}{H_{1}^{2}(t)} .
\end{aligned}
$$

Now summing the above expression from $t_{2}$ to $t-1$, we have

$$
\sum_{s=t_{2}}^{t-1} \Gamma(1-\alpha)<\sum_{s=t_{2}}^{t-1} \frac{\Delta H_{1}(s)}{H_{1}^{2}(s)}<\sum_{s=t_{2}}^{t-1}\left[\frac{H_{1}(s+1)}{H_{1}^{2}(s)}-\frac{H_{1}(s)}{H_{1}^{2}(s)}\right] .
$$

Since $\Delta H_{1}(t)$ is a non decreasing sequence, we have

$$
\sum_{s=t_{2}}^{t-1} \Gamma(1-\alpha)<\sum_{s=t_{2}}^{t-1}\left[\frac{1}{H_{1}(s+1)}-\frac{1}{H_{1}(s)}\right]<\frac{1}{H_{1}(t)}-\frac{1}{H_{1}\left(t_{2}\right)}<\frac{1}{H_{1}\left(t_{2}\right)} .
$$

Letting $t \rightarrow \infty, \lim _{t \rightarrow \infty} \sum_{s=t_{0}}^{t-1} \Gamma(1-\alpha)<\frac{1}{H_{1}\left(t_{2}\right)}$. This leads to a contradiction, which completes the proof of the theorem.

Theorem 7. Assume that $\left(C_{1}\right)$ to $\left(C_{3}\right)$ holds; and a positive double sequence $H(t, s)$ such that

$$
\begin{aligned}
& H(t, t)=0 \quad \text { for } t>t_{0} ; H(t, s)>0 \quad \text { for } t>s>t_{0} \\
& \Delta_{s} H(t, s)=H(t, s+1)-H(t, s) \leq 0 \text { for } t>s>t_{0} .
\end{aligned}
$$

If

$$
\lim _{t \rightarrow \infty} \operatorname{Sup} \frac{1}{H\left(t, t_{0}\right)} \sum_{s=t_{0}}^{t-1}\left[m q(s) H(t, s)-\frac{h_{+}^{2}(t, s) k}{4 \Gamma(1-\alpha) H(t, s)}\right]=\infty
$$

where $h_{+}(t, s)=\Delta_{s} H(t, s)-p(s) H(t, s)$, then every solution of equation (1) is oscillatory.

Proof. Suppose to the contrary that $u(t)$ is a non-oscillatory solution of (1). Without loss of generality, we take $u(t)$ is an eventually positive solution of (1). Proceeding as in Theorem - 6, we arrive at the equation (4). Now multiplying 
by $H(t, s)$ and taking summation from $t_{1}$ to $t-1$, we get

$$
\begin{aligned}
-\sum_{s=t_{1}}^{t-1} m q(s) H(t, s)>-\sum_{s=t_{1}}^{t-1} H(t, s) \Delta \omega(s) \\
\sum_{s=t_{1}}^{t-1}\left[\Gamma(1-\alpha) \omega^{2}(s) H(t, s)-p(s) \omega(s) H(t, s)\right] .
\end{aligned}
$$

Summation by parts formula yields

$$
-\sum_{s=t_{1}}^{t-1} H(t, s) \Delta \omega(s)=H\left(t, t_{1}\right) \omega\left(t_{1}\right)+\sum_{s=t_{1}}^{t-1} \omega(s+1) \Delta_{s} H(t, s) .
$$

Using equation (7) in (6) yields

$$
\begin{aligned}
-\sum_{s=t_{1}}^{t-1} m q(s) H(t, s) & >H\left(t, t_{1}\right) \omega\left(t_{1}\right)+\sum_{s=t_{1}}^{t-1} \omega(s+1) \Delta_{s} H(t, s)+ \\
& \sum_{s=t_{1}}^{t-1}\left[\Gamma(1-\alpha) \omega^{2}(s) H(t, s)-p(s) \omega(s) H(t, s)\right] .
\end{aligned}
$$

Since $\Delta \omega(t)>0$, we see that $\omega(t+1)>\omega(t)$ and we get

$$
\begin{gathered}
-\sum_{s=t_{1}}^{t-1} m q(s) H(t, s)>H\left(t, t_{1}\right) \omega\left(t_{1}\right)+ \\
\sum_{s=t_{1}}^{t-1}\left[\left(\Delta_{s} H(t, s)-p(s) H(t, s)\right) \omega(s)+\Gamma(1-\alpha) \omega^{2}(s) H(t, s)\right] \\
-\sum_{s=t_{1}}^{t-1} m q(s) H(t, s)>H\left(t, t_{1}\right) \omega\left(t_{1}\right)+ \\
\quad \sum_{s=t_{1}}^{t-1}\left[\Gamma(1-\alpha) H(t, s) \omega^{2}(s)+h_{+}(t, s) \omega(s)\right]
\end{gathered}
$$

where $h_{+}(t, s)=\Delta_{s} H(t, s)-p(s) H(t, s)$. Now

$$
\begin{aligned}
\sum_{s=t_{1}}^{t-1}\left[\Gamma(1-\alpha) H(t, s) \omega^{2}(s)+h_{+}(t, s) \omega(s)\right]=-\sum_{s=t_{1}}^{t-1} \frac{h_{+}^{2}(t, s)}{4 \Gamma(1-\alpha) H(t, s)} \\
+\sum_{s=t_{1}}^{t-1}\left[\sqrt{\Gamma(1-\alpha) H(t, s)} \omega(s)+\frac{h_{+}(t, s)}{2 \sqrt{\Gamma(1-\alpha) H(t, s)}}\right]^{2}
\end{aligned}
$$




$$
\sum_{s=t_{1}}^{t-1}\left[\Gamma(1-\alpha) H(t, s) \omega^{2}(s)+h_{+}(t, s) \omega(s)\right]>-\sum_{s=t_{1}}^{t-1} \frac{h_{+}^{2}(t, s)}{4 \Gamma(1-\alpha) H(t, s)}
$$

Using the above equation in (8), we get the following inequality

$$
\begin{aligned}
& -\sum_{s=t_{1}}^{t-1} m q(s) H(t, s)>H\left(t, t_{1}\right) \omega\left(t_{1}\right)-\sum_{s=t_{1}}^{t-1} \frac{h_{+}^{2}(t, s) k}{4 \Gamma(1-\alpha) H(t, s)} \\
& -H\left(t, t_{1}\right) \omega\left(t_{1}\right)>\sum_{s=t_{1}}^{t-1}\left[m q(s) H(t, s)-\frac{h_{+}^{2}(t, s) k}{4 \Gamma(1-\alpha) H(t, s)}\right] \\
& \sum_{s=t_{1}}^{t-1}\left[m q(s) H(t, s)-\frac{h_{+}^{2}(t, s) k}{4 \Gamma(1-\alpha) H(t, s)}\right]<H\left(t, t_{0}\right) \omega\left(t_{1}\right) .
\end{aligned}
$$

Since $\Delta_{s} H(t, s) \leq 0$ for $t>s \geq t_{0}$ and $0<H\left(t, t_{1}\right) \leq H\left(t, t_{0}\right)$ for $t>s \geq t_{0}$. Also $0<H(t, s) \leq H\left(t, t_{0}\right)$ for $t>s \geq t_{0}$, then $0<\frac{\bar{H}(t, s)}{H\left(t, t_{0}\right)} \leq 1$ for $t>s \geq t_{0}$. Hence

$$
\begin{gathered}
\frac{1}{H\left(t, t_{0}\right)} \sum_{s=t_{0}}^{t-1}\left[m q(s) H(t, s)-\frac{h_{+}^{2}(t, s) k}{4 \Gamma(1-\alpha) H(t, s)}\right] \leq \\
\frac{1}{H\left(t, t_{0}\right)} \sum_{s=t_{0}}^{t_{1}-1}\left[m q(s) H(t, s)-\frac{h_{+}^{2}(t, s) k}{4 \Gamma(1-\alpha) H(t, s)}\right]+ \\
\frac{1}{H\left(t, t_{0}\right)} \sum_{s=t_{1}}^{t-1}\left[m q(s) H(t, s)-\frac{h_{+}^{2}(t, s) k}{4 \Gamma(1-\alpha) H(t, s)}\right] .
\end{gathered}
$$

Using (9) in the above inequality, we obtain

$$
\begin{aligned}
\frac{1}{H\left(t, t_{0}\right)} \sum_{s=t_{0}}^{t-1}\left[m q(s) H(t, s)-\frac{h_{+}^{2}(t, s) k}{4 \Gamma(1-\alpha) H(t, s)}\right] \leq \\
\frac{1}{H\left(t, t_{0}\right)} \sum_{s=t_{0}}^{t_{1}-1}\left[m q(s) H(t, s)-\frac{h_{+}^{2}(t, s) k}{4 \Gamma(1-\alpha) H(t, s)}\right]+\omega\left(t_{1}\right) \\
\frac{1}{H\left(t, t_{0}\right)} \sum_{s=t_{0}}^{t-1}\left[m q(s) H(t, s)-\frac{h_{+}^{2}(t, s) k}{4 \Gamma(1-\alpha) H(t, s)}\right]<\omega\left(t_{1}\right)+\sum_{s=t_{0}}^{t_{1}-1} m q(s)
\end{aligned}
$$


Letting $t \rightarrow \infty$, we get

$$
\begin{aligned}
\lim _{t \rightarrow \infty} \operatorname{Sup} \frac{1}{H\left(t, t_{0}\right)} \sum_{s=t_{0}}^{t-1} & {\left[m q(s) H(t, s)-\frac{h_{+}^{2}(t, s) k}{4 \Gamma(1-\alpha) H(t, s)}\right] } \\
& <\omega\left(t_{1}\right)+\sum_{s=t_{0}}^{t_{1}-1} m q(s)<\infty
\end{aligned}
$$

which is a contradiction to (5). This completes the proof.

\section{References}

[1] A. George Maria Selvam, M. Paul Loganathan, R. Janagaraj and D. Abraham Vianny, Oscillation of fractional difference equations with damping terms, JP Journal of Mathematical Sciences, 16, No 1 (2016), 1-13.

[2] A. George Maria Selvam, M. Reni Sagayaraj, M. Paul Loganathan, Oscillatory behavior of a class of fractional difference equations with damping, International J. of Applied Mathematical Research, 3, No 3 (2014), 220224 .

[3] F.M. Atici, P.W. Eloe, Initial value problems in discrete fractional calculus, Proc. of the American Mathematical Society, 137, No 3 (2009), 981-989.

[4] A. Secera, H. Adiguzel, Oscillation of solutions for a class of nonlinear fractional difference equations, J. Nonlinear Sci. Appl., 9 (2016), 58625869 .

[5] E. Tunc, O. Tunc, On the oscillation of a class of Damped fractional differential equations, Miskolc Mathematical Notes, 17, No 1 (2016), 647-656.

[6] V. Muthulakshmi and S. Pavithra, Oscillatory behavior of fractional differential equation with damping, International $J$. of Mathematics and its Applications, 5, No 4-c (2017), 383-388.

[7] S.R. Grace, R.P. Agarwal, J.Y.P. Wong, A. Zafer, On the oscillation of fractional differential equations, Fractional Calculus and Applied Analysis, 15, No 2 (2012), 222-231.

[8] R.P. Agarwal, M. Bohner, S.R. Grace, D. O'Regan, Discrete Oscillation Theory, Hindawi Publishing Corporation, New York, NY 10022, USA, (2005). 
[9] Wei Nian Li, Oscillation results for certain forced fractional difference equations with damping term, Advances in Difference Equations, 2016 (2016), Art. 70, 9 pages. 
